\title{
Poisson structure and invariant manifolds on Lie groups
}

\author{
Jerrold E. Marsden \\ CDS, Caltech \\ Pasadena, CA 91125 USA \\ email: marsden@cds.caltech.edu
}

\author{
Sergey Pekarsky \\ CDS, Caltech \\ Pasadena, CA 91125 USA \\ email: pekarsky@cds.caltech.edu
}

\author{
Steve Shkoller \\ University of California \\ Davis, CA 95616 \\ email: shkoller@math.ucdavis.edu
}

International Conference on Differential Equations, Berlin, 1999

Edited by B. Fiedler, K. Gröger and J. Sprekels,

World Scientific, 2000, 1192-1197.

\begin{abstract}
For a discrete mechanical system on a Lie group $G$ determined by a (reduced) Lagrangian $\ell$ we define a Poisson structure via the pull-back of the Lie-Poisson structure on $\mathfrak{g}^{*}$ by the corresponding Legendre transform. The main result shown in this paper is that this structure coincides with the reduction under the symmetry group $G$ of the canonical discrete Lagrange 2 -form $\omega_{\mathbb{L}}$ on $G \times G$. Its symplectic leaves then become dynamically invariant manifolds for the reduced discrete system.
\end{abstract}

\section{Introduction}

Background. For systems on finite dimensional Lie groups $G$ with Lagrangians $L: T G \rightarrow \mathbb{R}$ that are $G$-invariant, discrete analogues of Euler-Poincaré and Lie-Poisson reduction theory (see, for example, Marsden and Ratiu [MR 99]) were developed in [MPeS 98]. The resulting discrete equations provide "reduced" numerical algorithms which manifestly preserve the symplectic structure. The manifold $G \times G$ is used as the discrete approximation of $T G$, and a discrete Lagrangian $\mathbb{L}: G \times G \rightarrow \mathbb{R}$ is constructed in such a way that the $G$-invariance property is preserved. Reduction by $G$ results in a new "variational" principle for the reduced Lagrangian $\ell: G \rightarrow \mathbb{R}$, which then determines the discrete Euler-Poincaré (DEP) equations. Reconstruction of these equations is consistent with the usual Veselov discrete Euler-Lagrange equations developed in [WM 97, MPS 98], which are naturally symplectic-momentum algorithms. Furthermore, the solution of the DEP algorithm leads directly to a discrete Lie-Poisson (DLP) algorithm. For the reader's benefit we summarize main results of the discrete reduction in the Appendix.

Motivation. Discretization of an Euler-Poincaré system on $T G$ results in a system on $G \times G$ defined by a Lagrangian $\mathbb{L}$. If it is regular, the Legendre transformations $F \mathbb{L}$ define a symplectic form (and, hence, a Poisson structure) on $\Delta \subset G \times G$ via the pull-back of the canonical form from $T^{*} G$. Then, general Poisson reduction applied to these discrete settings defines a Poisson structure on the reduced space $\mathcal{U}:=\pi_{d}(\Delta) \subset G$. This approach was adopted in Theorem 2.2 of [MPeS 98].

Alternatively, without appealing to the reduction procedure, a Poisson structure on a Lie group can be defined using ideas of Weinstein [W 96] on Lagrangian mechanics on groupoids and their algebroids. The key idea can be summarized in the following statements. A smooth function on a groupoid defines a natural (Legendre type) transformation between the groupoid and the dual of its algebroid. This transformation can be used to pull back a canonical Poisson structure from the dual of the algebroid, provided the regularity conditions are satisfied.

\section{Dynamics on groupoids and algebroids}

We briefly summarize results from Weinstein [W 96] and refer the reader to the original paper for details of proofs and definitions. Let $\Gamma$ be a groupoid over a set $M$, with $\alpha, \beta: \Gamma \rightarrow M$ being its source and 
target maps, with a multiplication map $m: \Gamma_{2} \rightarrow \Gamma$, where $\Gamma_{2} \equiv\{(g, h) \in \Gamma \times \Gamma \mid \beta(g)=\alpha(h)\}$. Denote its corresponding algebroid by $\mathcal{A}$.

The Lie groupoids relevant to our exposition are the Cartesian product $G \times G$ of a Lie group $G$, with multiplication $(g, h)(h, k)=(g, k)$, and the group $G$ itself. The corresponding algebroids are the tangent bundle $T G$ and the Lie algebra $\mathfrak{g}$, respectively. The dual bundle to a Lie algebroid carries a natural Poisson structure. This is the Poisson bracket associated to the canonical symplectic form on $T^{*} G$ and the Lie-Poisson structure on $\mathfrak{g}^{*}$, respectively.

Lagrangian mechanics on a groupoid $\Gamma$ is defined as follows. Let $\mathcal{L}$ be a smooth, real-valued function on $\Gamma, \mathcal{L}_{2}$ the restriction to $\Gamma_{2}$ of the function $(g, h) \mapsto \mathcal{L}(g)+\mathcal{L}(h)$.

Definition 2.1. Let $\Sigma_{\mathcal{L}} \subset \Gamma_{2}$ be the set of critical points of $\mathcal{L}_{2}$ along the fibers of the multiplication map $m$; i.e. the points in $\Sigma_{\mathcal{L}}$ are stationary points of the function $\mathcal{L}(g)+\mathcal{L}(h)$ when $g$ and $h$ are restricted to admissible pairs with the constraint that the product gh is fixed [W 96].

A solution of the Lagrange equations for the Lagrangian $\mathcal{L}$ is a sequence $\ldots, g_{-2}, g_{-1}, g_{0}, g_{1}, g_{2}, \ldots$ of elements of $\Gamma$, defined on some "interval" in $\mathbb{Z}$, such that $\left(g_{j}, g_{j+1}\right) \in \Sigma_{\mathcal{L}}$ for each $j$.

The Hamiltonian formalism for discrete Lagrangian systems is based on the fact that each Lagrangian submanifold of a symplectic groupoid determines a Poisson automorphism on the base Poisson manifold. Recall that the cotangent bundle $T^{*} \Gamma$ is, in addition to being a symplectic manifold, a groupoid itself, the base being $\mathcal{A}^{*}$; notice that both manifolds are naturally Poisson. The source and target mappings $\tilde{\alpha}, \tilde{\beta}: T^{*} \Gamma \rightarrow \mathcal{A}^{*}$ are induced by $\alpha$ and $\beta$.

Definition 2.2. Given any smooth function $\mathcal{L}$ on $\Gamma$, a Poisson map $\Lambda_{\mathcal{L}}$ from $\mathcal{A}^{*}$ to itself, which may be said to be generated by $\mathcal{L}$ is defined by the Lagrangian submanifold $d \mathcal{L}(\Gamma)$ (under a suitable hypothesis of nondegeneracy) [W 96].

The appropriate "Legendre transformation" $F \mathcal{L}$ in the groupoid context is given by $\tilde{\alpha} \circ d \mathcal{L}: \Gamma \rightarrow \mathcal{L}^{*}$ or $\tilde{\beta} \circ d \mathcal{L}: \Gamma \rightarrow \mathcal{L}^{*}$, depending on whether we consider right or left invariance (through the definition of maps $\tilde{\alpha}$ and $\tilde{\beta}$ ). The transformation $F \mathcal{L}$ relates the mapping on $\Gamma$ defined by $\Sigma_{\mathcal{L}}$ with the mapping $\Lambda_{\mathcal{L}}$ on $\mathcal{A}^{*} . F \mathcal{L}$ also pulls back the Poisson structure from $\mathcal{A}^{*}$ to $\Gamma$, which, in general, is defined only locally on some neighborhood $\mathcal{U} \subset \Gamma$. In the context of a Lie group, this means that any regular function $\ell: G \rightarrow \mathbb{R}$ defines a Poisson structure on $\mathcal{U}$. We shall address this issue in the next sections. The reader is referred to [W 96] for an application of the above ideas to the groupoid $M \times M$ when the manifold $M$ does not necessarily have group structure.

\section{$3 \quad$ DEP as generators of Lie-Poisson Hamilton-Jacobi equations}

A Lie group $G$ is the simplest example of a groupoid with the base being just a point. Its algebroid is the corresponding Lie algebra $\mathfrak{g}$, with the dual being $\mathfrak{g}^{*}$. Consider left invariance and let a general function $\mathcal{L}$ on the group be specified by the discrete reduced Lagrangian $\ell: G \rightarrow \mathbb{R}$. Then, the Legendre transform defined above is given by $F \ell=L_{g}^{*} \circ d \ell: G \rightarrow \mathfrak{g}^{*}$, where $d \ell: G \rightarrow T^{*} G$. Using these transformations we define $\Pi_{k-1} \equiv F \ell\left(f_{k k-1}\right)=L_{f_{k k-1}}^{*} \circ d \ell\left(f_{k k-1}\right)$. Recall the DEP equation (4.4) for left-invariant systems

: $L_{f_{k+1 k}}^{*} d \ell\left(f_{k+1 k}\right)-R_{f_{k-1}}^{*} d \ell\left(f_{k k-1}\right)=0$, where we have identified the notations $\ell^{\prime}$ and $d \ell$. The later equation can be rewritten as a system

$$
\left\{\begin{array}{l}
\Pi_{k}=L_{f}^{*} \circ d \ell(f) \\
\Pi_{k+1}=R_{f}^{*} \circ d \ell(f)
\end{array}\right.
$$

where the first equation is to be solved for $f$ (which stands for $f_{k+1 k}$ ) which then is substituted into the second equation to compute $\Pi_{k+1}$.

This system is precisely the Lie-Poisson Hamilton-Jacobi system described in [GM 88] with the reduced discrete Lagrangian $\ell$ playing the role of the generating function. This means that there is no need to find an approximate solution of the reduced Hamilton-Jacobi equation [GM 88]. Notice also that the DLP equation is a direct consequence of the system (3.1): $\Pi_{k+1}=\operatorname{Ad}_{f_{k+1 k}^{-1}}^{*} \cdot \Pi_{k}$. 
The following diagrams relate the dynamics on $G$ and on $\mathfrak{g}^{*}$ :
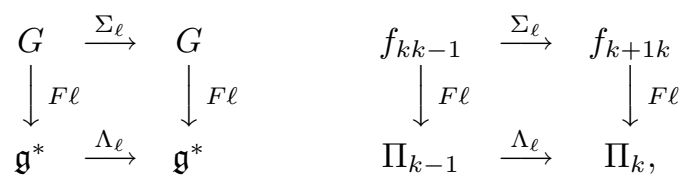

where $\Sigma_{\ell}$ and $\Lambda_{\ell}$ are given in Definitions 2.1 and 2.2.

\section{Some Advantages of Structure-preserving Integrators}

As we mentioned above, the "Legendre transform" $F \ell$ allows us to put a Poisson structure on the Lie group $G$, which, of course, depends on the discrete Lagrangian on $G \times G$, and hence on the original Lagrangian $L$ on $T G$ (if we consider this from the discrete reduction point of view). It follows that the reduction of the discrete Euler-Lagrange dynamics on $G \times G$ is necessarily restricted to the symplectic leaves of this Poisson structure, so that these leaves are invariant manifolds, and correspond (under $F \ell^{*}$ ) to the symplectic leaves (coadjoint orbits) of the continuous reduced system on $\mathfrak{g}^{*}$.

These ideas are the content of the following theorems. Here we state the theorems (for the case of right invariance) and only sketch their proofs. The reader is referred to [MPeS 99] for details. Analogous theorems hold for the case of left invariant systems.

Theorem 4.1. Let $L$ be a right invariant Lagrangian on $T G$ and let $\mathbb{L}$ be the Lagrangian of the corresponding discrete system on $\mathcal{U} \subset G \times G$. Assume that $\mathbb{L}$ is regular, in the sense that the Legendre transformation $F \mathbb{L}: \Delta \rightarrow F \mathbb{L}(\Delta) \subset T^{*} G$ is a local diffeomorphism, and let the quotient maps be given by

$$
\pi_{d}: G \times G \rightarrow(G \times G) / G \cong G \text { and } \pi: T^{*} G \rightarrow\left(T^{*} G\right) / G \cong \mathfrak{g}^{*} .
$$

Let $\ell$ be the reduced Lagrangian on $G$ defined by $\mathbb{L}=\ell \circ \pi_{d}$, and let $F \ell: G \rightarrow \mathfrak{g}^{*}$ be the corresponding Legendre transform. Then the following diagram commutes:

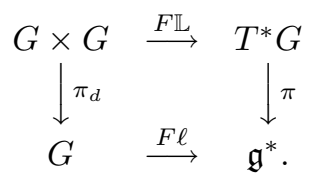

Idea of the proof. Choosing appropriate coordinate systems on each space, we can rewrite this diagram as follows:

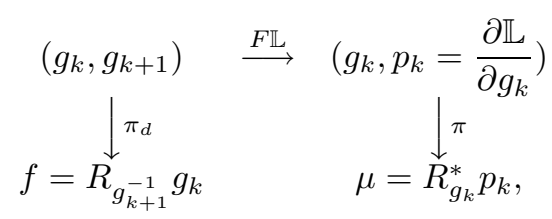

where $f$ stands for $f_{k k+1}=g_{k} g_{k+1}^{-1}$. To close this diagram and to verify the arrow determined by $F \ell$ compute the derivative of $\mathbb{L}$ using the chain rule and use definitions of the partial derivative $\partial f / \partial g_{k}$ and the Legendre transformation $F \ell$ :

$$
\mu=R_{g_{k}}^{*} p_{k}=R_{g_{k}}^{*} \frac{\partial(\ell \circ \pi)}{\partial g_{k}}=R_{g_{k}}^{*}\left(R_{g_{k+1}^{-1}}^{*} \frac{\partial \ell}{\partial f}\right)=R_{f}^{*} \frac{\partial \ell}{\partial f}=R_{f}^{*} \circ \ell^{\prime}(f),
$$

Corollary 4.1. Reconstruction of the discrete Lie-Poisson (DLP) dynamics on $\mathfrak{g}^{*}$ by $\pi^{-1}$ corresponds to the image of the discrete Euler-Lagrange (DEL) dynamics on $G \times G$ under the Legendre transformations $F \mathbb{L}$ and results in an algorithm on $T^{*} G$ approximating the continuous flow of the corresponding Hamiltonian system. 
Idea of the proof. The proof follows from the results of the previous section, in particular, diagram (3.2) relates the DLP dynamics on $\mathfrak{g}^{*}$ with the DEP dynamics on $\mathcal{U} \subset G$ which, in turn, is related to the DEL dynamics on $\Delta \subset G \times G$ via the reconstruction.

Theorem 4.2. The Poisson structure on the Lie group $G$ obtained by reduction of the Lagrange symplectic form $\omega_{\mathbb{L}}$ on $\Delta \subset G \times G$ via $\pi_{d}$ coincides with the Poisson structure on $\mathcal{U} \subset G$ obtained by the pull-back of the Lie-Poisson structure $\omega_{\mu}$ on $\mathfrak{g}^{*}$ by the Legendre transformation Fl. (see diagram (4.1) above).

The proof is based on the commutativity of the following diagrams
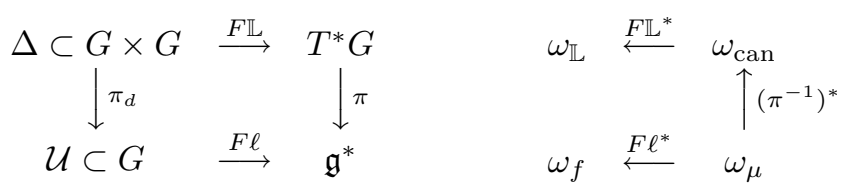

and the $G$ invariance of the unreduced symplectic forms.

\section{Discussions}

Main Results of this Paper. We show that when a discrete Lagrangian $\mathbb{L}: G \times G \rightarrow \mathbb{R}$ is $G$-invariant, a Poisson structure on (a subset) of one copy of the Lie group $G$ can be defined which governs the corresponding discrete reduced dynamics. The symplectic leaves of this structure become dynamically invariant manifolds which are manifestly preserved under the structure preserving discrete Euler-Poincaré algorithm.

We apply Weinstein's results on Lagrangian mechanics on groupoids and algebroids [W 96] to the setting of regular Lie groups. Then, starting with a discrete Euler-Poincaré system on $G$ one can readily recover, by means of the Legendre transformation, the corresponding Lie-Poisson Hamilton-Jacobi system on $\mathfrak{g}^{*}$ analyzed by Ge and Marsden [GM 88].

Various Important Remarks. First of all, we remark that the discrete symplectic structure $\omega_{\mathbb{L}}$ is not globally defined, but rather is only nondegenerate in a neighborhood $\Delta$ of the diagonal in $G \times G$, i.e. whenever $g_{k}$ and $g_{k+1}$ are nearby. It follows then that the reduced Poisson structure $\{f, h\}_{G}$ need only be defined on $\mathcal{U}$, where $\mathcal{U}$ is the image of $\Delta$.

An important remark to Corollary 4.1 which follows from the results in [KMO 99] is that, in general, to get a corresponding algorithm on the Hamiltonian side which is consistent with the corresponding continuous Hamiltonian system on $T^{*} G$, one must use the time step $h$-dependent Legendre transform given by the map

$$
\left(g_{k}, g_{k+1}\right) \mapsto\left(g_{k},-h D_{1} \mathbb{L}\left(g_{k}, g_{k+1}\right)\right) .
$$

The results of this paper are not effected, however, as we assume $h$ to be constant and so we would simply add a constant multiplier to the corresponding symplectic and Poisson structures. For variable time-stepping algorithms, this remark is crucial and must be taken into account.

More General Configuration Spaces or Where to Go. The ideas outlined in this paper carry over to the integration of systems defined on a general configuration space $M$ with some symmetry group $G$. In this case, the reduced discrete space $(M \times M) / G$ inherits a Poisson structure from the one defined on $M \times M$ (analogously to (4.5)). Its symplectic leaves again become dynamically invariant manifolds for structure-preserving integrators and can be viewed as images of the symplectic leaves of the reduced Poisson manifold $T^{*} M / G$ under appropriately defined "Legendre transformations".

The groupoid-algebroid formalism is very well suited to the discrete gauge field theory generalization as well as to discrete semi-direct product theory. The latter is related to the recent results of Bobenko and Suris [BS 98]. It would be very interesting to develop the semi-direct product point of view on the discrete level. The relation to Routhian reduction and how it can fit into the discrete semi-direct product theory should be further investigated. 
Last but not least, the groupoid-algebroid formalism can be used to define a Poisson structure on a Lie algebra $\mathfrak{g}$ using the duality between Lie-Poisson and Euler-Poincaré reduced systems on $\mathfrak{g}^{*}$ and $\mathfrak{g}$, respectively. A reduced Lagrangian $l$ determines the Legendre transformations $F l$ from $\mathfrak{g}$ to $\mathfrak{g}^{*}$ and its pull-back $F l^{*}$ defines Casimirs on $\mathfrak{g}$ by $C_{\mathfrak{g}}(\xi)=F l^{*} \cdot C_{\mathfrak{g}^{*}}(\xi)$. Besides purely theoretical interest, this can have applications for the analysis of dynamics on Lie algebras.

\section{Acknowledgments}

The authors would like to thank Alan Weinstein for pointing out the connections with the general theory of dynamics on groupoids and algebroids.

\section{Appendix: Discrete Reduction}

In this appendix we review the discrete Euler-Poincaré reduction of a Lagrangian system on $G \times G$ considered in detail in [MPeS 98]. See [V 88, V 91, WM 97, MPS 98, LS 96, BS 98] for various related aspects of discrete mechanics. We approximate $T G$ by $G \times G$ and form a discrete Lagrangian $\mathbb{L}$ : $G \times G \rightarrow \mathbb{R}$ from the original Lagrangian $L$ on $T G$. We choose discretization schemes for which the discrete Lagrangian $\mathbb{L}$ inherits the symmetries of the original Lagrangian $L: \mathbb{L}$ is $G$-invariant on $G \times G$ whenever $L$ is $G$-invariant on $T G$. In particular, the induced right (left) lifted action of $G$ onto $T G$ corresponds to the diagonal right (left) action of $G$ on $G \times G$. Then, application of discrete variational principle results in discrete Euler-Lagrange (DEL) equations as well as the discrete symplectic form $\omega_{\mathbb{L}}$

The discrete Euler-Poincaré algorithm. The discrete reduction of a right-invariant system proceeds as follows. The induced group action on $G \times G$ by an element $\bar{g} \in G$ is simply right multiplication in each component: $\bar{g}:\left(g_{k}, g_{k+1}\right) \mapsto\left(g_{k} \bar{g}, g_{k+1} \bar{g}\right)$, for all $g_{k}, g_{k+1} \in G$. (Of course, some systems such as the rigid body are left invariant.)

The quotient map is given by $\pi_{d}: G \times G \rightarrow(G \times G) / G \cong G, \quad\left(g_{k}, g_{k+1}\right) \mapsto g_{k} g_{k+1}^{-1}$. We note that one may alternatively use $g_{k+1} g_{k}^{-1}$ instead of $g_{k} g_{k+1}^{-1}$ as the quotient map; this alternative choice is used in [BS 98]. The projection map defines the reduced discrete Lagrangian $\ell: G \rightarrow \mathbb{R}$ for any $G$-invariant $\mathbb{L}$ by $\ell \circ \pi_{d}=\mathbb{L}$, so that $\ell\left(g_{k} g_{k+1}^{-1}\right)=\mathbb{L}\left(g_{k}, g_{k+1}\right)$

A reduced discrete variational principle results in the discrete Euler-Poincaré (DEP) equations

$$
R_{f_{k k+1}}^{*} \ell^{\prime}\left(f_{k k+1}\right)-L_{f_{k-1 k}}^{*} \ell^{\prime}\left(f_{k-1 k}\right)=0
$$

for $k=1, \ldots, N-1$, where $R_{f}^{*}$ and $L_{f}^{*}$ are the right and left pull-backs by $f$, respectively, and $\ell^{\prime}$ : $G \rightarrow T^{*} G$ is the differential of $\ell$ defined as follows. Let $g^{\epsilon}$ be a smooth curve in $G$ such that $g^{0}=g$ and $\left.(d / d \epsilon)\right|_{\epsilon=0} g^{\epsilon}=v$, then $\ell^{\prime}(g) \cdot v=\left.(d / d \epsilon)\right|_{\epsilon=0} \ell\left(g^{\epsilon}\right)$. In the case that $\mathbb{L}$ is left invariant, the discrete Euler-Poincaré equations take the form

$$
L_{f_{k+1 k}}^{*} \ell^{\prime}\left(f_{k+1 k}\right)-R_{f_{k k-1}}^{*} \ell^{\prime}\left(f_{k k-1}\right)=0
$$

where $f_{k+1 k} \equiv g_{k+1}^{-1} g_{k}$ is in the left quotient $(G \times G) / G$.

The symplectic structure $\omega_{\mathbb{L}}$ naturally defines a Poisson structure on $\Delta \subset G \times G$ (which we shall denote $\left.\{\cdot, \cdot\}_{G \times G}\right)$ by the relation $\{F, H\}_{G \times G}=\omega_{\mathbb{L}}\left(X_{F}, X_{H}\right)$. Then, Theorem 2.2 of [MPeS 98] states that if the action of $G$ on $G \times G$ is proper, the algorithm on $G$ defined by the discrete Euler-Poincaré equations (4.3) preserves the induced Poisson structure $\{\cdot, \cdot\}_{G}$ on $\mathcal{U} \subset G$ given by

$$
\{f, h\}_{G} \circ \pi_{d}=\left\{f \circ \pi_{d}, h \circ \pi_{d}\right\}_{G \times G}
$$

for any $C^{1}$ functions $f, h$ on $\mathcal{U}$, where $\mathcal{U}=\pi_{d}(\Delta)$.

Reconstruction. Using the definition $f_{k k+1}=g_{k} g_{k+1}^{-1}$, the DEL algorithm can be reconstructed from the DEP algorithm by

$$
\left(g_{k-1}, g_{k}\right) \mapsto\left(g_{k}, g_{k+1}\right)=\left(f_{k-1 k}^{-1} \cdot g_{k-1}, f_{k k+1}^{-1} \cdot g_{k}\right)
$$


where $f_{k k+1}$ is the solution of (4.3). Indeed, $f_{k k+1}^{-1} \cdot g_{k}$ is precisely $g_{k+1}$. Similarly one shows that in the case of a left $G$ action, the reconstruction of the DEP equations (4.4) is given by $\left(g_{k-1}, g_{k}\right) \mapsto\left(g_{k}, g_{k+1}\right)=$ $\left(g_{k-1} \cdot f_{k k-1}^{-1}, g_{k} \cdot f_{k+1 k}^{-1}\right)$.

The discrete Lie-Poisson algorithm In addition to reconstructing the dynamics on $\Delta \subset G \times G$, one may use the coadjoint action to form a discrete Lie-Poisson algorithm approximating the dynamics on $\mathfrak{g}^{*}[\mathrm{MPeS} 98]$

$$
\mu_{k+1}=\operatorname{Ad}_{f_{k k+1}}^{*} \cdot \mu_{k}
$$

where $\mu_{k}:=\operatorname{Ad}_{g_{k}^{-1}}^{*} \mu_{0}, \mu_{0}$ is the constant of motion (the momentum map value), and the sequence $\left\{f_{k k+1}\right\}$ is provided by the DEP algorithm on $G$. The corresponding discrete Lie-Poisson equations for

the left invariant system is given by $\Pi_{k+1}=\operatorname{Ad}_{f_{k+1 k}^{-1}}^{*} \cdot \Pi_{k}$, where $\Pi_{k}:=\operatorname{Ad}_{g_{k}}^{*} \pi_{0}$ and $\pi_{0}$ is the constant momentum map.

\section{References}

[AK 98] V.I. ARnold and B. Khesin, Topological Methods in Hydrodynamics, Springer Verlag, New York, 1998.

[BS 98] A.I. Bobenko ANd Yu.B. SuRIs Discrete time Lagrangian mechanics on Lie groups, with an application to the Lagrange top, preprint.

[GM 88] Z.Ge and J.E. Marsden, Lie-Poisson Hamilton-Jacobi theory and Lie-Poisson integrators, Phys. Lett A, 133, (1988), 134-139.

[KMO 99] C. Kane, J.E. Marsden, and M. Ortiz, Symplectic-energy-momentum preserving variational integrators, to appear in J. Math. Physics.

[LS 96] D. Lewis and J.C. Simo, Conserving algorithms for the $N$ dimensional rigid body, Fields. Inst. Comm., 10, (1996), 121-139.

[MPS 98] J.E. Marsden, G. Patrick, and S. Shkoller, Multisymplectic geometry, variational integrators, and nonlinear PDEs, Comm. Math. Phys., 199, (1998), 351-395.

[MPeS 98] J.E. Marsden, S. Pekarsky, and S. Shkoller, Discrete Euler-Poincaré and Lie-Poisson Equations, to appear in Nonlinearity.

[MPeS 99] J.E. Marsden, S. Pekarsky, and S. Shkoller, Symmetry Reduction of Discrete Lagrangian Mechanics on Lie groups, submitted to $C M P$.

[MR 99] J.E. Marsden And T.S. RATiu, Introduction to Mechanics and Symmetry, Springer-Verlag, 1994. Second Edition, 1999.

[MoV 91] J. Moser And A.P. Veselov, Discrete versions of some classical integrable systems and factorization of matrix polynomials, Comm. Math. Phys., 139, (1991), 217-243.

[V 88] A.P. Veselov, Integrable discrete-time systems and difference operators, Funk. Anal. Prilozhen., 22, (1988), 1-13.

[V 91] A.P. Veselov, Integrable Lagrangian correspondences and the factorization of matrix polynomials, Funk. Anal. Prilozhen., 25, (1991), 38-49.

[W 96] A. Weinstein, Lagrangian mechanics and groupoids, Fields. Inst. Comm., 7, (1996), 207-231.

[WM 97] J.M. Wendlandt and J.E. Marsden, Mechanical integrators derived from a discrete variational principle, Physica D, 106, (1997), 223-246. 